\title{
Mitral Valve Surgery in a Patient 50 Years after a Pneumonectomy
}

\author{
Hartwig H. C. von Oehsen ${ }^{1}$ Benjamin A. Högerle ${ }^{20}$ \\ ${ }^{1}$ Department of Public Health, Saarlouis Municipal Council, Saarland, \\ Germany \\ ${ }^{2}$ Department of Thoracic Surgery, Thoraxklinik at Heidelberg \\ University Hospital, Heidelberg, Germany \\ ${ }^{3}$ Department of Thoracic and Cardiovascular Surgery, Saarland \\ University Medical Center, Faculty of Medicine, Saarland University, \\ Homburg, Saarland, Germany \\ Thorac Cardiovasc Surg Rep 2019;8:e14-e17.
}

Christian Giebels $^{3}$ Hans-Joachim Schäfers ${ }^{3}$

\begin{abstract}
Keywords

- heart failure

- mitral valve surgery

- thoracic surgery
\end{abstract}

\author{
Address for correspondence Benjamin A. Högerle, MD, Department \\ of Thoracic Surgery, Thoraxklinik at Heidelberg University Hospital, \\ Röntgenstraße 1, Heidelberg 69126, Germany \\ (e-mail: benjamin.hoegerle@med.uni-heidelberg.de).
}

\section{Background}

Pneumonectomy is primarily performed to address central lung cancer. However, survival after the procedure is limited by the aggressiveness of the tumor disease. Long-term survivors were frequent in the last century when pneumonectomy was more widely used in, for example, the treatment of tuberculosis. ${ }^{1}$ Long-term complications include pulmonary hypertension, a decrease in lung function, and heart failure, possibly due to the aberrant cardiac axis., ${ }^{2,3}$ Reports of extensive long-term heart failure in patients decades after a pneumonectomy are rare (using MEDLINE/ PubMed, search terms: pneumonectomy, valve reconstruction/regurgitation/replacement). Although cardiac surgery and reoperations become necessary, they are complex to manage because of the challenging anatomical conditions and the impaired clinical status of the patient.

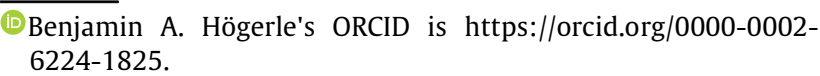

received

January 20, 2019

accepted after revision

April 1, 2019
For the first time, this case report describes the medical history of a patient who underwent heart valve surgery three times after pneumonectomy due to a recurrent heart failure. Furthermore, the treatment strategy and the surgical technique are presented. Both are critically discussed retrospectively.

\section{Case Description}

The patient was diagnosed with a malignant testicular tumor in 1964. A unilateral orchiectomy was performed. In 1968, left-sided pulmonary metastases were detected, and a pneumonectomy was performed due to the extent of the tumor. The patient was recovered well. The further oncological follow-up showed no recurrence of the malignancy, and postoperative lung function remained stable.

An increase in mitral valve (MV) regurgitation was observed starting in 1987 (-Fig. 1). Despite optimal medical therapy, the physical performance of the patient was deteriorated over time (up to New York Heart Association [NYHA] class IV). In 2000, the clinical symptoms were worsened, and

\footnotetext{
(C) 2019 Georg Thieme Verlag KG Stuttgart · New York
}

License terms

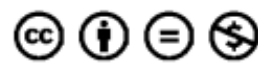




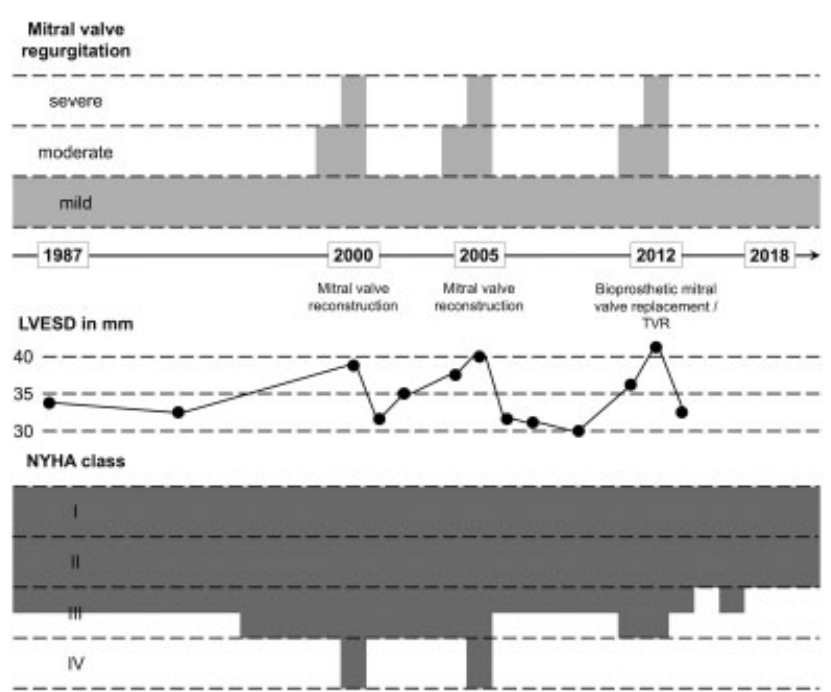

Fig. 1 A chronological overview from 1987 to 2018. The patient underwent a pneumonectomy in 1968. In 1987, the first clinical symptoms were manifested due to increasing mitral valve regurgitation. Mitral valve reconstructions were performed in 2000 and 2005. In 2012, the patient received a simultaneous bioprosthetic mitral valve replacement and a TVR. The left ventricular end-systolic diameter is displayed over time, which correlates with the regurgitation. The physical performance is represented by the NYHA classification. LVESD, left ventricular end-systolic diameter in mm; NYHA, New York Heart Association; TVR, tricuspid valve reconstruction.

pulmonary edema was occurred. Echocardiography showed severe MV regurgitation due to an anterior mitral leaflet (AML) prolapse with preserved left ventricular systolic function. The patient underwent extensive cardiorespiratory tests for preoperative risk assessment. The maximal oxygen uptake $\left(\mathrm{VO}_{2} \max \right)$ was $13 \mathrm{~mL} \mathrm{O} / \mathrm{kg} / \mathrm{min}$. The case was discussed by the heart team and the patient was considered as suitable for surgery. MV reconstruction was performed using a median sternotomy and a standard cannulation for cardiopulmonary bypass and cardioplegic arrest. The AML prolapse was repaired using artificial chords. An annuloplasty ring was implanted.

After an initial positive result, regurgitation was recurred. New episodes of atrial fibrillation (AF) required antiarrhythmic medication and cardioversion. In 2005, relevant recurrent MV regurgitation was diagnosed again in the presence of severe heart failure symptoms (NYHA class IV). Again, $\mathrm{VO}_{2} \max \left(13 \mathrm{~mL} \mathrm{O}_{2} / \mathrm{kg} / \mathrm{min}\right)$ and all other cardiorespiratory tests confirmed that the patient was suitable for surgery. A redo MV reconstruction was performed using a median sternotomy; additional artificial chords were implanted. Ablation of the left atrium was performed by unipolar high-frequency electricity. At that time, the posterior part of the mitral annulus appeared deformed through the descending aorta.

Postoperatively, mild residual regurgitation was found, and systolic left ventricular dimensions were decreased. The physical performance of the patient was improved (NYHA class II/III). Recurrent episodes of AF were treated chemically and by cardioversion. Finally, long-term sinus rhythm was achieved.
Since 2011, the clinical symptoms recurred progressively. In 2012, severe MV regurgitation with mildly increased left ventricular size was noted. In addition, moderate tricuspid valve regurgitation and a permanent AF were observed. In addition to the basic cardiorespiratory tests, this time, it was not possible to perform extensive exercise tests due to the impaired clinical status of the patient. A 6-minute walk test was performed instead. A need for supplemental oxygen ( $3 \mathrm{~L} /$ $\mathrm{min}$ ) was detected after $300 \mathrm{~m}$. As the lung function was still adequate, the additional oxygen supply was explained by the impaired cardiac function. The case was discussed by the heart team. A combined MV reconstruction and tricuspid valve reconstruction were recommended in light of the following facts: (1) besides the heart, all other functional parameters were acceptable; (2) the patient had no other relevant comorbidities; and (3) the patient had recovered well after all three previous thoracic surgeries.

During the subsequent redo surgery (via a median sternotomy), it was not possible to achieve an adequate leaflet configuration of the AML. A bioprosthetic MV replacement was performed, and the tricuspid annulus was reduced. During the procedure, adhesions and an aberrant cardiac axis after pneumonectomy were present but manageable. Postoperatively, the patient developed pneumonia, which required intravenous antibiotics and a prolonged stay in the intensive care unit. The patient was recovered very well. A pacemaker was implanted using interventional cardiology due to a permanent AF. Temporarily, an additional oxygen supply of $1 \mathrm{~L} / \mathrm{min}$ was necessary.

In 2015, endocarditis affecting the bioprosthetic MV occurred. This was likely related to a dental treatment in combination with an antibiotic resistance to the standard prophylaxis. The antibiotics were adjusted, and the inflammation was decreased.

Six years after the latest cardiac surgery, and 50 years after the pneumonectomy, only a mild MV regurgitation is present. No relevant tricuspid regurgitation is present. There is no need for treatment of the known mild pulmonary hypertension (mean pulmonary arterial pressure [MPAP] 1990: 12 mm Hg, mPAP 2005: 39 mm Hg, and mPAP 2015: 27 mm $\mathrm{Hg}$ ). The patient is in good physical shape (NYHA class II) and requires no additional oxygen supply.

\section{Discussion}

The first valve replacement after a pneumonectomy was reported by Izzat et al in $1995 .{ }^{4} \mathrm{~A}$ patient received a mechanical MV replacement 10 years after a right-sided pneumonectomy due to a bronchial carcinoid tumor. Imoto et al presented a case with 41-year period between rightsided pneumonectomy and MV replacement, which is the longest period to date. ${ }^{5}$ Based on a MEDLINE/PubMed search, only 18 valve reconstructions or replacements after pneumonectomies have been reported. No case studies have looked at redo heart valve surgery after a pneumonectomy.

To date, Stoller et al have provided the most detailed review. ${ }^{6}$ That study evaluated 19 patients who received coronary artery bypass grafts or heart valve surgery 

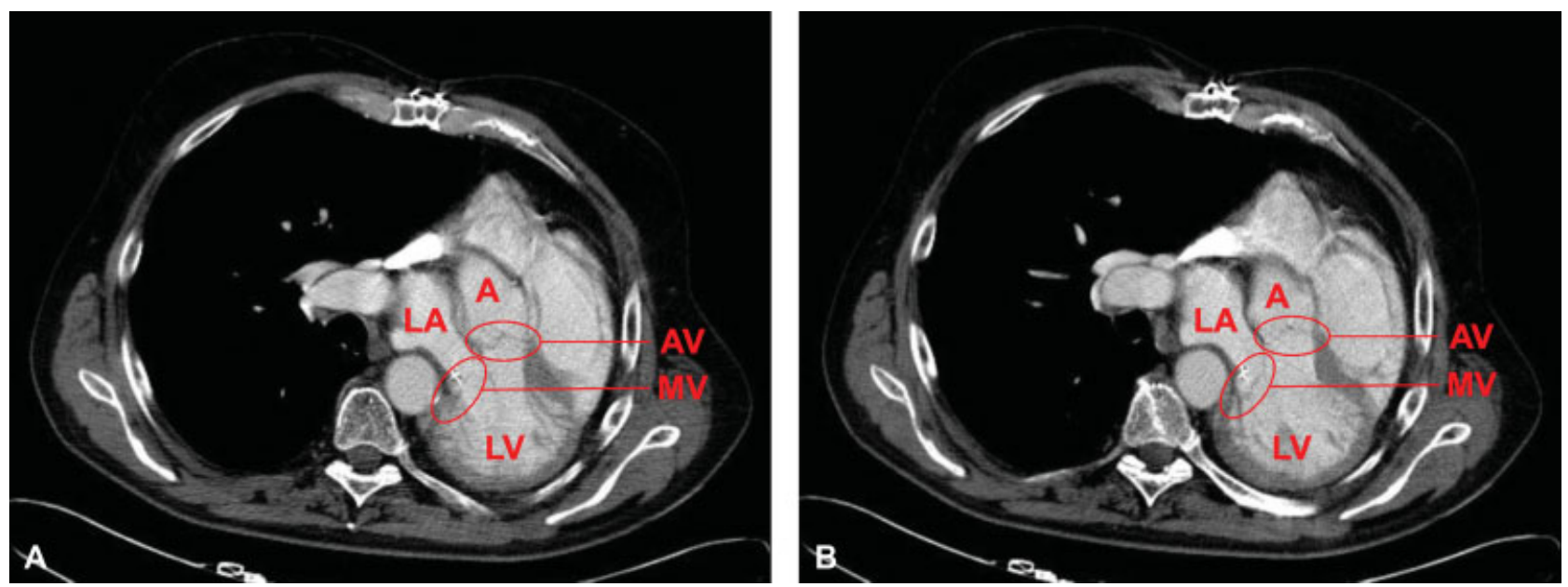

Fig. 2 Preoperative computed tomography of the thorax recorded in 2006 after the second mitral valve reconstruction. The heart is rotated in the left hemithorax after a left pneumonectomy. Due to this mediastinal shift, median sternotomy offers the possibility of optimal mitral valve exposure. A, aorta; AV, aortic valve; LA, left atrium; LV, left ventricle; MV, mitral valve.

following prior pneumonectomy. Only one MV reconstruction and four valve replacements were performed in 40 years during the period under review. At that time, all thoracic surgeries after pneumonectomies were high-risk procedures because the risk of postoperative complications was $50 \%$, and the overall mortality rate was $16 \%$.

Thoracic surgeries after a pneumonectomy are high-risk procedures because patients have a mediastinal shift (-Fig. 2). This leads to aberrant anatomical conditions, which often make treatment options difficult, including those offered by interventional cardiology. In general, an edge-toedge MitraClip and newer strategies used to reduce the annulus dilatation (e.g., Carillion) are available for transcatheter MV repair. Moreover, several transcatheter MV replacement systems have recently been developed (e.g., Tendyne). ${ }^{7}$ However, to date, no cases of interventional MV reconstruction or replacement postpneumonectomy have been described. This might be due to the altered position of the heart, which complicates the interventional implantation of the devices in the correct position. Therefore, while surgery is also difficult, the results are often more predictable. For the same reasons, interventional treatment options were excluded by the heart team in the current case.

Most heart valve surgeries were performed after right-sided pneumonectomies. The long-term effects of pneumonectomy on the position of the heart are characterized by a rotation of the heart in patients after left-sided pneumonectomy. In contrast, a lateral shift is often observed after right-sided pneumonectomy. ${ }^{3}$ The surgical strategy must consider the existing anatomy with marked mediastinal distortion, the compression of the left atrium by the descending aorta due to the total displacement of the heart to the left side, the option for cannulation to establish extracorporeal circulation, and the exposure of the target area. Especially, after a right-sided pneumonectomy, minimally invasive approaches to the MV should be considered. ${ }^{8,9}$ Otherwise, a median sternotomy is recommended. In cases of extreme mediastinal displacement, an anterior thoracotomy is preferred. ${ }^{10}$ The most suitable surgical approach should be discussed after using a cardiac computed tomography. ${ }^{11}$ In the current case, the median sternotomy provided perfect exposure of the MV due to the rotation of the heart into the left chest. It remains unclear whether the possible interference between the descending aorta and the MV led to the distortion of the mitral annulus. This may have contributed to the recurrence of mitral regurgitation.

AML prolapse is the main mechanism underlying mitral regurgitation. ${ }^{12}$ In the current case, prolapse was corrected in 2000 by chordal replacement and implantation of an annuloplasty ring. After intraoperative assessment, the recurrent mitral regurgitation, which led to a subsequent surgery in 2005 , was apparently caused by recurrent AML prolapse. The size of the MV annulus was still normal. However, the posterior part of the MV annulus appeared to be deformed by the close local relationship to the descending aorta. The AML prolapse was corrected again using simple additional chordal replacement until the valve had a good configuration under cardioplegic cardiac arrest. This course underlines the difficulty of rerepairing the anterior leaflet with neochordae. Retrospectively, the influence of the altered configuration of the MV annulus by the descending aorta may have been underestimated in this case. After implantation of a flexible annuloplasty ring, the annular stabilization under these anatomical circumstances, and later under dynamic conditions, may not have been sufficient. Thus, in retrospect, in 2005 MV replacement would have been the preferable procedure.

Apart from the operation, the perioperative management can be challenging in single lung patients. Preoperatively, the altered position of the heart can lead to difficulties in standard diagnostic tests. In the current case, the equivalent of the apical echocardiographic views was obtained in a left paravertebral position. For preoperative evaluation, vital capacity, forced expiratory volume in 1 second, and diffusing capacity of the lung for carbon monoxide may be appropriate parameters to determine a patient's suitability for heart surgery. ${ }^{13}$ Due to the small number of cases, to date, no 
standard values have been published; thus, the heart team should thoroughly discuss all diagnostic tests on a case-bycase basis. Furthermore, lung volumes are reduced postpneumonectomy. Restrictive ventilatory defects often occur. In contrast, the remaining lung is hyperinflated. ${ }^{14}$ During surgery, mechanical ventilation settings must be adjusted accordingly. Postoperatively, any compromise of ventilatory function (e.g., pneumonia) can become a life-threatening complication. Besides, in the current case, it became evident that the patient had developed antimicrobial resistance to the standard prophylaxis over time. This resulted in an endocarditis affecting the bioprosthetic MV. The possibility of resistance to antibiotics should therefore be considered to avoid such complications in similar patients.

In conclusion, this case report underlines that heart valve surgeries after pneumonectomies (and redo surgeries) are feasible even under complex conditions. In case of relevant heart valve disease and when no other treatment options are available, patients may benefit from surgery. However, only a few studies on this topic have been published. More data are required to develop a standardized approach to identify adequate treatment strategies for these patients.

\section{Authors' Contribution}

Hartwig H. C. von Oehsen collected and analyzed the data. Benjamin A. Högerle analyzed the data and wrote the case report. Christian Giebels provided and analyzed additional data. Hans-Joachim Schäfers was the surgeon in charge. All authors revised the case report for intellectual content and participated until the final approval of the submitted version. All authors read and approved the final case report.

\section{Conflict of Interest}

None.

\section{Acknowledgment}

The authors would like to thank Rainer Breit, MD (Department of Cardiology and Pneumology, Merzig Hospital, Merzig, Germany) for his great personal commitment.

\section{References}

1 Subotic D, Yablonskiy P, Sulis G, et al. Surgery and pleuropulmonary tuberculosis: a scientific literature review. J Thorac Dis 2016;8(07):E474-E485

2 Deslauriers J, Ugalde P, Miro S, et al. Long-term physiological consequences of pneumonectomy. Semin Thorac Cardiovasc Surg 2011;23(03):196-202

3 Smulders SA, Holverda S, Vonk-Noordegraaf A, et al. Cardiac function and position more than 5 years after pneumonectomy. Ann Thorac Surg 2007;83(06):1986-1992

4 Izzat MB, Regragui IA, Angelini GD. Mitral valve replacement after previous right pneumonectomy. Ann Thorac Surg 1995;59(01): 222-224

5 Imoto Y, Sese A, Sakamoto M, Tanaka K, Matsumoto T, Kajiwara T. [Mitral valve replacement 41 years after right pneumonectomy]. Kyobu Geka 2001;54(13):1087-1090

6 Stoller JK, Blackstone E, Pettersson G, Mihaljevic T. Coronary artery bypass graft and/or valvular operations following prior pneumonectomy: report of four new patients and review of the literature. Chest 2007;132(01):295-301

7 Lavall D, Hagendorff A, Schirmer SH, Böhm M, Borger MA, Laufs U. Mitral valve interventions in heart failure. ESC Heart Fail 2018;5 (04):552-561

8 Rose D, Liew CK, Zacharias J. Mitral valve repair after a right pneumonectomy: a minimally invasive approach. Interact Cardiovasc Thorac Surg 2015;21(04):551-553

9 Sinha S, Morgan-Hughes N, O'Toole L, Hunter S. Minimal access mitral valve repair in a patient with a right pneumonectomy for Scimitar syndrome. Interact Cardiovasc Thorac Surg 2016;22(06): 851-853

10 Barreda T, Laali M, Dorent R, Acar C. Left thoracotomy for aortic and mitral valve surgery in a case of mediastinal displacement due to pneumonectomy. J Heart Valve Dis 2008;17 (02):239-242

$11 \mathrm{Kim} \mathrm{JH}$, Kim EY, Jin GY, Choi JB. A review of the use of cardiac computed tomography for evaluating the mitral valve before and after mitral valve repair. Korean J Radiol 2017;18(05):773-785

12 Pfannmüller B, Seeburger J, Davierwala P, Mohr FW. Repair of the anterior mitral leaflet prolapse. Expert Rev Med Devices 2014;11 (01):89-100

13 Mutlak H, Czerner S, Winter H, Zwissler B, Lackermeier P. [Preoperative evaluation and risk estimation in thoracic surgery]. Anaesthesist 2010;59(10):918-928

14 Luzzi L, Tenconi S, Voltolini L, et al. Long-term respiratory functional results after pneumonectomy. Eur J Cardiothorac Surg 2008;34(01):164-168 